\title{
Transverse Single-Spin Asymmetries in Proton-Proton Collisions at the AFTER@LHC Experiment
}

\author{
K. Kanazawa, ${ }^{1}$ Y. Koike, ${ }^{2}$ A. Metz, ${ }^{1}$ and D. Pitonyak ${ }^{3}$ \\ ${ }^{1}$ Department of Physics, SERC, Temple University, Philadelphia, PA 19122, USA \\ ${ }^{2}$ Department of Physics, Niigata University, Ikarashi, Niigata 950-2181, Japan \\ ${ }^{3}$ RIKEN BNL Research Center, Brookhaven National Laboratory, Upton, NY 11973, USA
}

Correspondence should be addressed to D. Pitonyak; dpitonyak@quark.phy.bnl.gov

Received 9 March 2015; Accepted 24 April 2015

Academic Editor: Jean-Philippe Lansberg

Copyright (C) 2015 K. Kanazawa et al. This is an open access article distributed under the Creative Commons Attribution License, which permits unrestricted use, distribution, and reproduction in any medium, provided the original work is properly cited. The publication of this article was funded by SCOAP $^{3}$.

We present results for transverse single-spin asymmetries in proton-proton collisions at kinematics relevant for AFTER, a proposed fixed-target experiment at the Large Hadron Collider. These include predictions for pion, jet, and direct photon production from analytical formulas already available in the literature. We also discuss specific measurements that will benefit from the higher luminosity of AFTER, which could help resolve an almost 40-year puzzle of what causes transverse single-spin asymmetries in proton-proton collisions.

\section{Introduction}

Transverse single-spin asymmetries (TSSAs), denoted by $A_{N}$, have been a fundamental observable since the mid-1970s to test perturbative quantum chromodynamics (pQCD). Such measurements were first conducted at FermiLab, where large effects were found in $p B e \rightarrow \Lambda^{\uparrow} X$ [1]. These results contradicted the naïve collinear parton model, which said that $A_{N}$ should be extremely small [2], and doubts were raised as to whether pQCD can actually describe these reactions [2]. However, in the 1980s it was shown that if one went beyond the parton model and included collinear twist-3 (CT3) quark-gluon-quark correlations in the nucleon, substantial TSSAs could be generated [3, 4]. In the 1990s this CT3 approach was worked out in more detail for protonproton collisions, first for direct photon production [5-7] and then for pion production [8]. Over the last decade, several other analyses furthered the development of this formalism; see [9-19] and references therein. During the same time, another mechanism was also put forth to explain TSSAs in proton-proton collisions. This approach involves the Sivers [20, 21], Collins [22], and Boer-Mulders [23, 24] transverse momentum dependent (TMD) functions and became known as the Generalized Parton Model (GPM); see [25-29] and references therein. (We mention that since most likely a rigorous factorization formula involving TMD functions does not hold for single-inclusive processes (which have only one scale), the GPM can only be considered a phenomenological model.) In addition to all of this theoretical work, many experimental measurements of $A_{N}$ have been performed at proton-(anti)proton accelerators [30-42]. Most of the experimental data in the more negative $x_{F}$ region has come in the form of light-hadron asymmetries $A_{N}^{h}$, for example, $h=\pi, K, \eta$, with the exception of the jet asymmetry $A_{N}^{\text {jet }}$ measured a few years ago at the Relativistic Heavy Ion Collider (RHIC) by the $\mathrm{A}_{N}$ DY Collaboration [40]. (Throughout the paper we will use the convention $x_{F}=$ $2 l_{z} / \sqrt{S}$, where $l$ is the momentum of the outgoing particle, and the transversely polarized proton moves along the $-z$ axis. That is, $x_{F} \rightarrow-1$ means large momentum fractions $x^{\uparrow}$ of the parton probed inside the transversely polarized proton. This setup causes $x_{F}$ to be opposite in sign to the one used in collider experiments (like those at RHIC).) Plans are also in place to measure the direct photon asymmetry $A_{N}^{\gamma}$ at RHIC by both the PHENIX Collaboration and the STAR Collaboration [43-45].

Although much progress has been made in understanding TSSAs, there is not a definitive answer on what their 
origin might be. In the CT3 approach it was assumed for many years that a soft-gluon pole (SGP) chiral-even quarkgluon-quark (qgq) matrix element called the Qiu-Sterman (QS) function $T_{F}(x, x)$ was the main cause of $A_{N}^{\pi}[8,11]$. However, this led to a so-called "sign-mismatch" between the QS function and the TMD Sivers function $f_{1 T}^{\perp}$ extracted from semi-inclusive deep-inelastic scattering (SIDIS) [46]. This issue could not be resolved through more flexible parameterizations of the Sivers function [47]. Moreover, the authors of [48] argued, by looking at $A_{N}$ data on the target TSSA in inclusive DIS $[49,50]$, that $T_{F}(x, x)$ cannot be the main source of $A_{N}^{\pi}$. This observation led us last year in [51] to analyze $A_{N}^{\pi}$ by including not only the QS function but also the fragmentation mechanism, whose analytical formula was first fully derived in [18] (the so-called "derivative term" was first computed in [52]) (see also [17, 53, 54] for fragmentation terms in other processes). We found in this situation for the first time in $\mathrm{pQCD}$ that one can fit all RHIC high transverse momentum pion data very well without any signmismatch issue. Furthermore, we showed that a simultaneous description of TSSAs in $p^{\uparrow} p \rightarrow \pi X$, SIDIS, and $e^{+} e^{-} \rightarrow$ $h_{1} h_{2} X$ is possible. Nevertheless, more work must be done to confirm/refute this explanation and its predictions. We mention that, in the GPM, one cannot draw a definitive conclusion as to whether the Sivers or Collins mechanism is the main cause of $A_{N}^{\pi}[28,29]$. (In principle the Boer-Mulders function and gluon Sivers function can also contribute to the GPM formalism, but these pieces have not been analyzed in the literature.) This is due to the theoretical error bands being too large, since the associated TMD functions are mostly unconstrained in the large $-x^{\uparrow}$ regime covered by the data $[28,29]$. For a detailed discussion of the GPM formalism and its predictions for the AFTER experiment, see [55].

In addition, in order to have a complete knowledge of TSSAs, it is important to have a "clean" extraction of the QS function from observables like $A_{N}^{\text {jet }}$ and $A_{N}^{\gamma}$ that do not have any fragmentation contributions. (We will ignore photons coming from fragmentation [56], which can be largely suppressed by using isolation cuts.) (For recent analyses of $A_{N}^{\gamma}$ in $p^{\uparrow} A$ collisions, see $[57,58]$.) This is necessary in order to help resolve the sign-mismatch issue and better understand the role of rescattering effects in the nucleon. The jet asymmetry has been studied in $[11,46,59,60]$ and the direct photon asymmetry has been investigated in $[6,11,12,56,59-64]$. It is important to point out that other contributions to $A_{N}^{\text {jet }}$ and $A_{N}^{\gamma}$ exist besides the one from the (SGP $q 9 q$ chiral-even) $\mathrm{QS}$ function. These other pieces include (i) soft-fermion pole (SFP) chiral-even qgq functions, (ii) SGP and SFP qgq chiralodd functions, and (iii) SGP trigluon functions. For $A_{N}^{\gamma}$ the numerical analyses in $[59,64]$ show that (i) is negligible for $x_{F}<0$ while the study in [64] draws a similar conclusion for (ii) as does the work in [62] for (iii). That is, for $A_{N}^{\gamma}$, the QS function dominates the asymmetry. We mention that, at present in the GPM, $A_{N}^{\gamma}$ is predicted to have the opposite sign to that from the CT3 approach [29]. Therefore, as was emphasized in [64], this observable could allow us for the first time to clearly distinguish between the two frameworks as well as learn about the process dependence of the Sivers function [65], which is a feature of this nonperturbative object that is crucial to our current understanding of TMD functions.

For $A_{N}^{\text {jet }}$ the conclusions as to which piece dominates are not as clear. The study in $[66,67]$ provides evidence that (ii) should be small in the whole $x_{F}$-region. The work in [59] shows that the same is most likely true for (i) but that analysis suffers from the sign-mismatch issue. Also, in [19] there is an indication that (iii) could be significant. Therefore, it will be necessary to reassess the impact of (i) and (iii) on $A_{N}^{\text {jet }}$. Nevertheless, one can gain insight into these other terms by looking at the contribution from the QS function and comparing it with data.

Given the open issues that still remain, it is an opportune time for the Large Hadron Collider (LHC) to produce data on TSSAs in proton-proton collisions via the AFTER experiment. These measurements will not only add to the data from FermiLab, AGS, and RHIC but also, through the high luminosity of the experiment $[68,69]$, probe certain features that remain ambiguous. For example, the behavior of $A_{N}^{\pi}$ at large pion transverse momentum $l_{T}$ appears to fall off very slowly (or is even flat), a feature which the theory says should persist to high $l_{T}[29,51,55,59]$ (see also [70] in the context of $\Lambda^{\uparrow}$ production). However, the data from RHIC [71] has too large error bars (or not enough statistics) in this high- $l_{T}$ region to ascertain whether or not this is true. Also, $A_{N}^{\text {jet }}$ measured by $\mathrm{A}_{N} \mathrm{DY}$ [40] has large error bars as $x_{F}$ becomes more negative, which makes it difficult to determine whether or not the QS function alone can describe that data. Moreover, as previously mentioned, $A_{N}^{\gamma}$ has never been measured before, yet it could be a tremendous opportunity to learn about the process dependence of the Sivers function and distinguish between the CT3 and GPM frameworks. Already, PHENIX and STAR plan to carry out such experiments [4345].

Therefore, in this paper we give predictions within the CT3 formalism for $A_{N}^{\pi}, A_{N}^{\text {jet }}$, and $A_{N}^{\gamma}$ at AFTER@LHC kinematics. (For related work on charmonium and bottomonium production we refer to $[72,73]$.) Since the relevant analytical formulas already exist within the literature, in Section 2 we focus on the phenomenology and refer the reader to the appropriate papers on the underlying theory. These numerical results are summarized in Section 3, and there we highlight again how AFTER can offer unique insight into TSSAs in proton-proton collisions, which is a truly fundamental observable to test $\mathrm{PQCD}$ at higher twist.

\section{Pion, Photon, and Jet TSSAs at AFTER}

We start first with $A_{N}^{\pi}$, where we follow our numerical work in [51]. (We also refer the reader to [11, 13, 18] for more formal discussions of the relevant analytical formulas.) There we took into account the contribution from the QS function and the fragmentation term. The former has a model-independent relation to the Sivers function [74], while the latter involves three nonperturbative CT3 fragmentation functions (FFs): $\widehat{H}, \widehat{H}_{F U}^{\mathfrak{I}}$, and $H$. Of these, $\widehat{H}$ has a modelindependent connection to the Collins function $[18,52]$ 


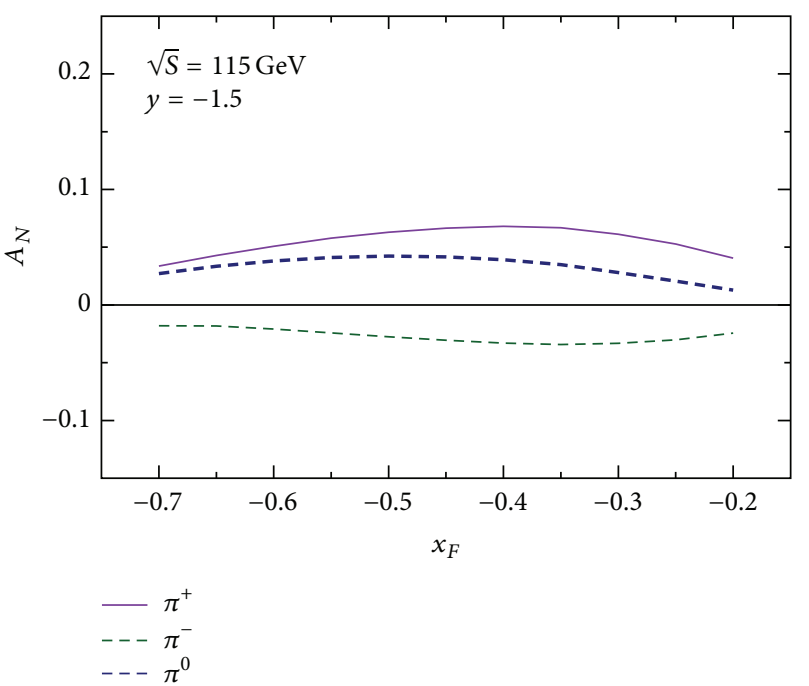

(a)

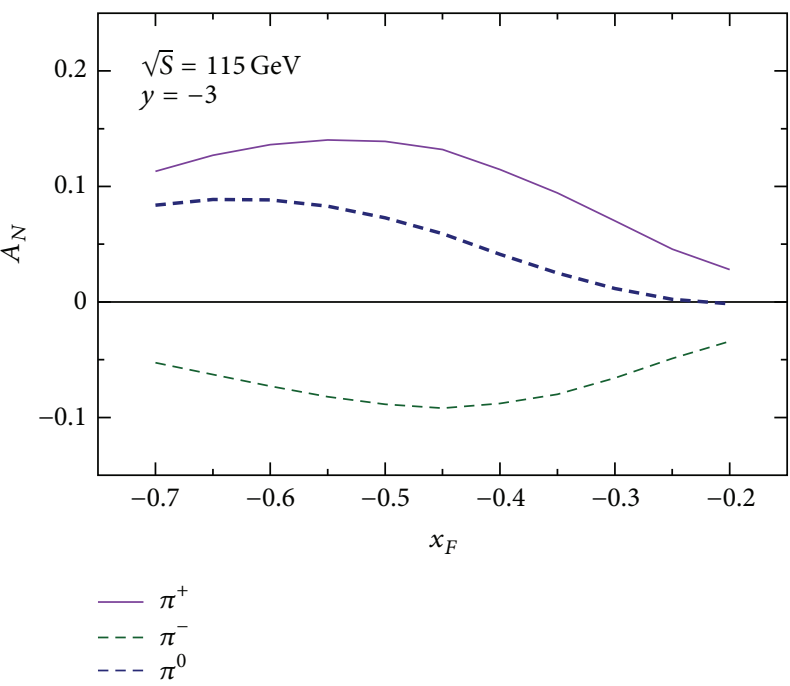

(b)

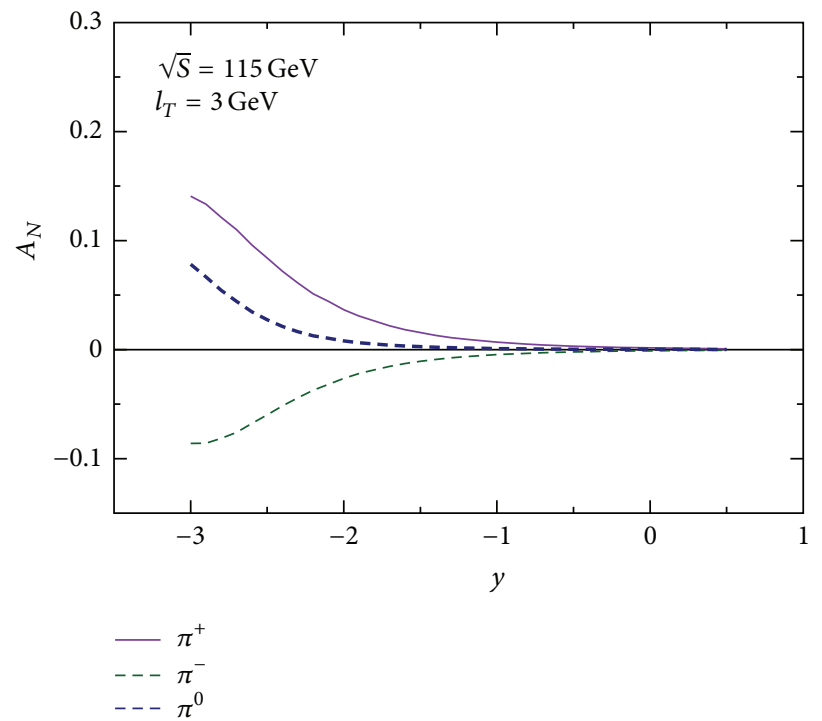

(c)

Figure 1: $A_{N}^{\pi}$ versus $x_{F}$ at fixed $y=-1.5$ (a) and $y=-3$ (b) and $A_{N}^{\pi}$ versus $y$ at fixed $l_{T}=3 \mathrm{GeV}$ (c). All plots are at $\sqrt{S}=115 \mathrm{GeV}$ for pion production at AFTER.

and $H$ can be written in terms of the other two through a QCD equation-of-motion relation [18]. In Figures 1 and 2 we provide predictions for neutral and charged pion production at AFTER based on our fit in [51]. One sees in Figure 1 from $A_{N}^{\pi}$ versus $x_{F}$ that the magnitude of the asymmetry can be anywhere from $\sim 5-10 \%$ and from $A_{N}^{\pi}$ versus $y$ that it increases with more negative (center-of-mass) rapidity $y$. (Recall the relation between $x_{F}, y$, and $l_{T}: x_{F}=$ $2 l_{T} \sinh (y) / \sqrt{S}$, so $A_{N}$ versus $x_{F}\left(A_{N}\right.$ versus $\left.y\right)$ at fixed $y\left(l_{T}\right)$ implies a running in $l_{T}\left(x_{F}\right)$.) One also notices that $A_{N}^{\pi}$ turns over at more negative $x_{F}$ values, which was also observed in some of the STAR data $[33,34,39]$. In Figure 2, where we show $A_{N}^{\pi}$ versus $l_{T}$, one sees that the asymmetry is flat or falls off very slowly as $l_{T}$ increases, a feature that had also been measured by STAR [71]. It will be important to establish with more precision if this flatness persists at higher- $l_{T}$ values, say $12-15 \mathrm{GeV}$, and AFTER, with its much higher luminosity, will be in a position to make such a measurement.

We next look at $A_{N}^{\text {jet }}$ and $A_{N}^{\gamma}$, which do not receive contributions from FFs. As we discussed in Section 1, the former may receive nonnegligible contributions from terms other than the QS function, while for the latter we recently showed in [64] that the QS function is the dominant piece to that asymmetry. (All of the analytical expressions for $A_{N}^{\gamma}$ can be found in [64] while those for $A_{N}^{\text {jet }}$ are determined simply by setting $D_{1}(z)$ (the unpolarized FF) to $\delta(1-z)$ in the equations for $A_{N}^{\pi}$ given in $[11,14,19,66,67]$.) (We note the $A_{N}^{\gamma}$ analytical formulas for the piece involving chiral-odd functions are new from [64], while those involving chiral-even functions were derived before in the literature, and the relevant references are 


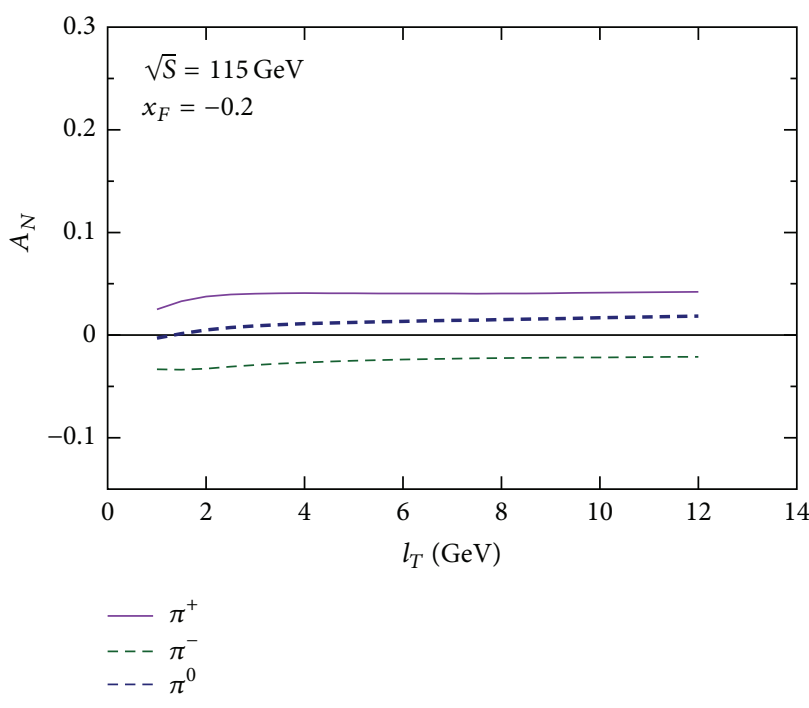

(a)

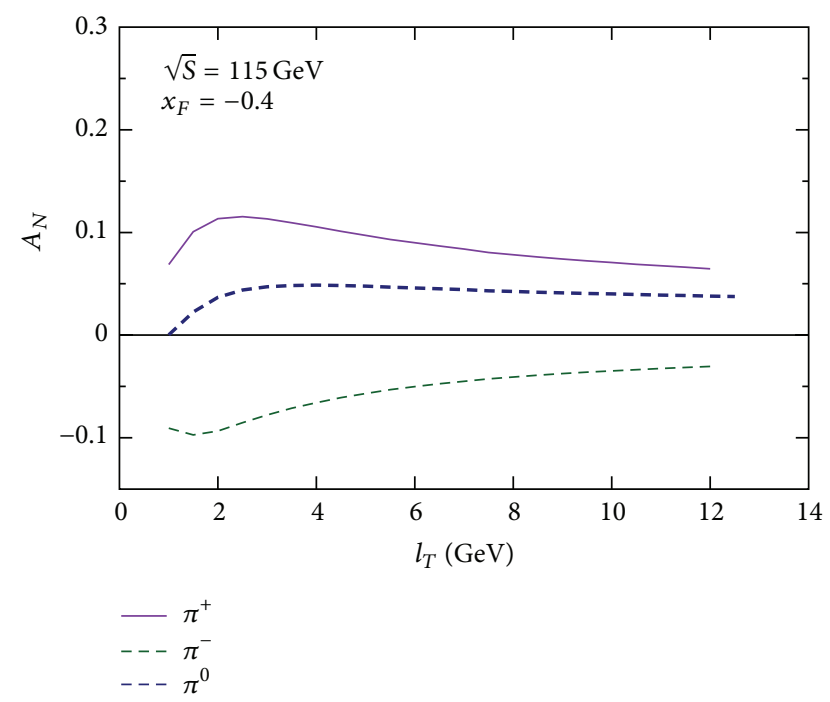

(b)

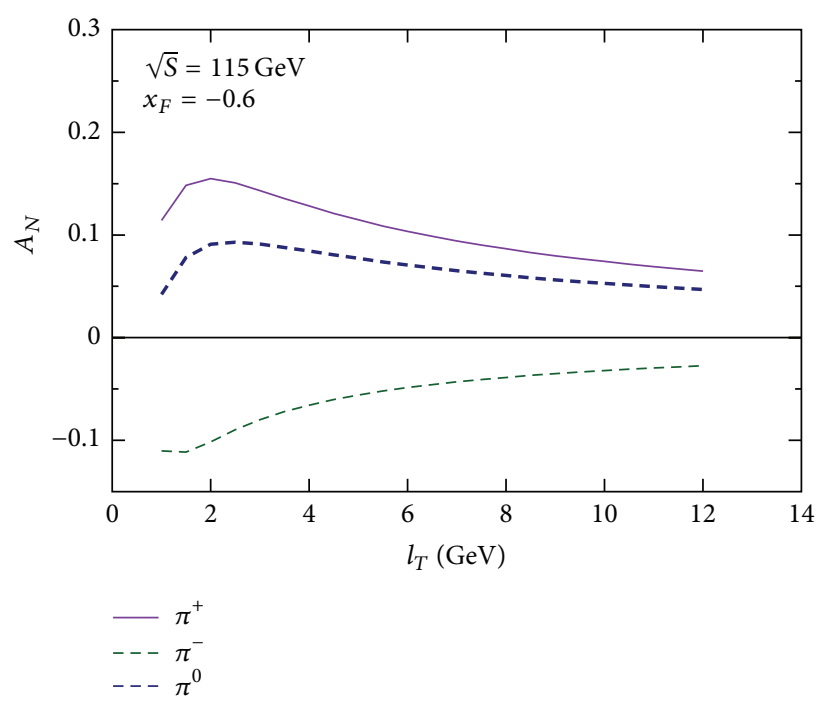

(c)

Figure 2: $A_{N}^{\pi}$ versus $l_{T}$ at fixed $x_{F}=-0.2(\mathrm{a}), x_{F}=-0.4(\mathrm{~b})$, and $x_{F}=-0.6(\mathrm{c})$ at $\sqrt{S}=115 \mathrm{GeV}$ for pion production at AFTER.

cited therein.) However, given that the other pieces for $A_{N}^{\text {jet }}$ are not reliably known, for that asymmetry we will only look at the contribution from the QS function using its relation to the Sivers function, while for $A_{N}^{\gamma}$ we adopt our work in [64]. In Figures 3 and 4 we show results for jet and photon production at AFTER. We see that $A_{N}^{\text {jet }}$ is very small, although we caution the reader that the Sivers function (which we use as input for the QS function) is mostly unconstrained in the large- $x^{\uparrow}$ region, and when this uncertainty is taken into account, one could obtain a measurable asymmetry [60]. Also, as we mentioned, there is the potential for (chiral-even) SFP and/or trigluon functions to make an impact. Therefore, in order to determine if the Sivers function alone can describe $A_{N}^{\text {jet}}$, along with the current data from $\mathrm{A}_{N} \mathrm{DY}$, we need more precise data in the far backward region, which should be possible at AFTER. (We note that STAR has preliminary data on electromagnetic "jets" that could also be helpful [75].)

Unlike the jet asymmetry, $A_{N}^{\gamma}$ could be on the order of $\sim-5 \%$ at less negative $x_{F}$ and more negative $y$ (see Figures $3(\mathrm{a})$ and $3(\mathrm{~b})$ ) or smaller $l_{T}$ and less negative $x_{F}$ (see Figure 4). Both of these observations are consistent with the behavior of $A_{N}^{\gamma}$ as a function of rapidity (see Figure 3(c)), where the asymmetry peaks at $y \sim-2$ (with $l_{T}=3 \mathrm{GeV}$ ), which corresponds to $x_{F} \sim-0.2$. Since the QS function is the dominant source of the asymmetry, we can have "clean" access to it. We state again that the GPM framework at present predicts $A_{N}^{\gamma}$ to be positive [29]. Therefore, a clear nonzero signal for this observable would help to distinguish between the CT3 and GPM formalisms. However, we emphasize that should data contradict the predictions of the GPM, this does not invalidate the results obtained for TMD observables that 


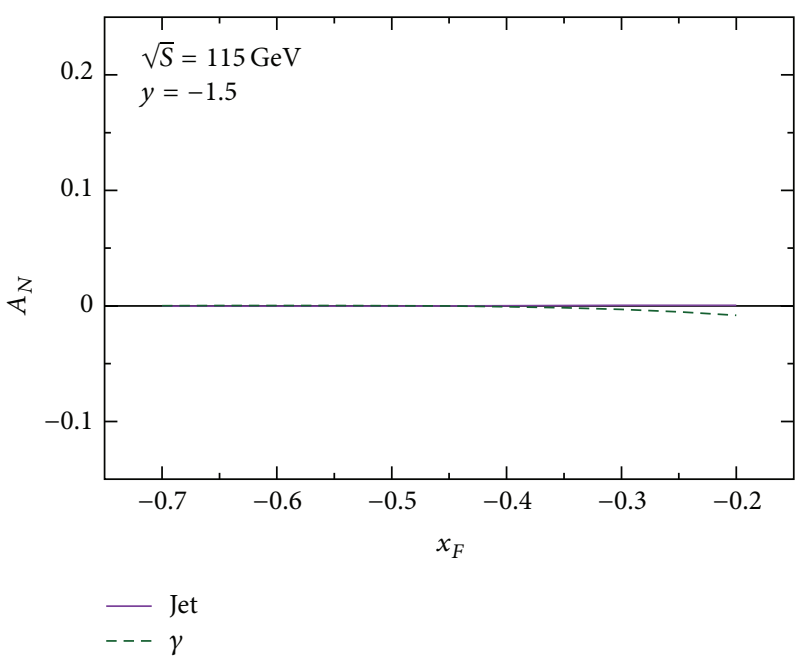

(a)

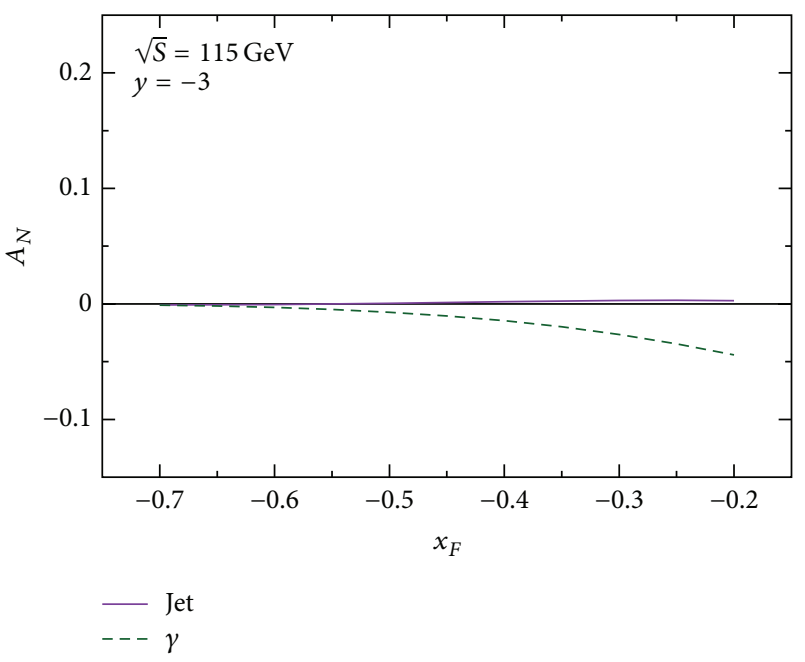

(b)

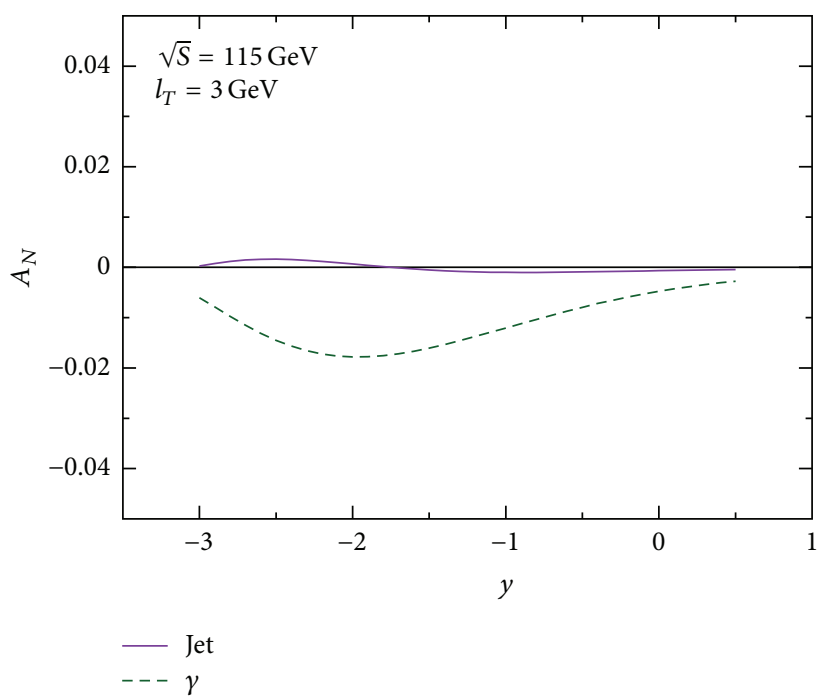

(c)

Figure 3: $A_{N}$ versus $x_{F}$ at fixed $y=-1.5$ (a) and $y=-3$ (b) and $A_{N}$ versus $y$ at fixed $l_{T}=3 \mathrm{GeV}$ (c). All plots are at $\sqrt{S}=115 \mathrm{GeV}$ for jet/photon production at AFTER.

are based on rigorous TMD factorization proofs. Also, since we use the Sivers function from SIDIS as our input for the QS function, we can learn about the predicted process dependence of the Sivers function.

\section{Summary and Outlook}

In this paper we have discussed TSSAs in single-inclusive pion, jet, and photon production from proton-proton collisions, that is, $p^{\uparrow} p \rightarrow\{\pi$, jet, $\gamma\} X$, at kinematics relevant for the proposed AFTER@LHC experiment. These asymmetries have been fundamental observables to test $\mathrm{pQCD}$ at higher twist for close to 40 years, and much work has been performed on both the theoretical and experimental sides. Nevertheless, issues still remain as to the origin of these TSSAs, which makes a measurement of $A_{N}$ at the LHC via the AFTER experiment timely. For $A_{N}^{\pi}$ we have found that AFTER should expect (absolute) asymmetries on the order of $5-10 \%$ as a function of $x_{F}$ and increasing as the rapidity becomes more negative. Also, the $l_{T}$ dependence of $A_{N}^{\pi}$ still falls off slowly and flattens out at high $l_{T}$. For $A_{N}^{\text {jet }}$ we predict a very small asymmetry, but we must remember that uncertainties in the Sivers function could allow for a measurable observable [60] and also that other contributions (like chiral-even SFP and trigluon) could make an impact. Lastly, for $A_{N}^{\gamma}$ we expect asymmetries on the order of $\sim-5 \%$ and decreasing with more negative $x_{F}$ and increasing $l_{T}$. These are opposite in sign to the ones predicted from the GPM [29].

Even though these observables have been (or are planned to be) measured at RHIC, AFTER has the ability, through its much higher luminosity, to not only supplement the RHIC data but also provide important information on still unknown issues. For example, it will be key to determine 


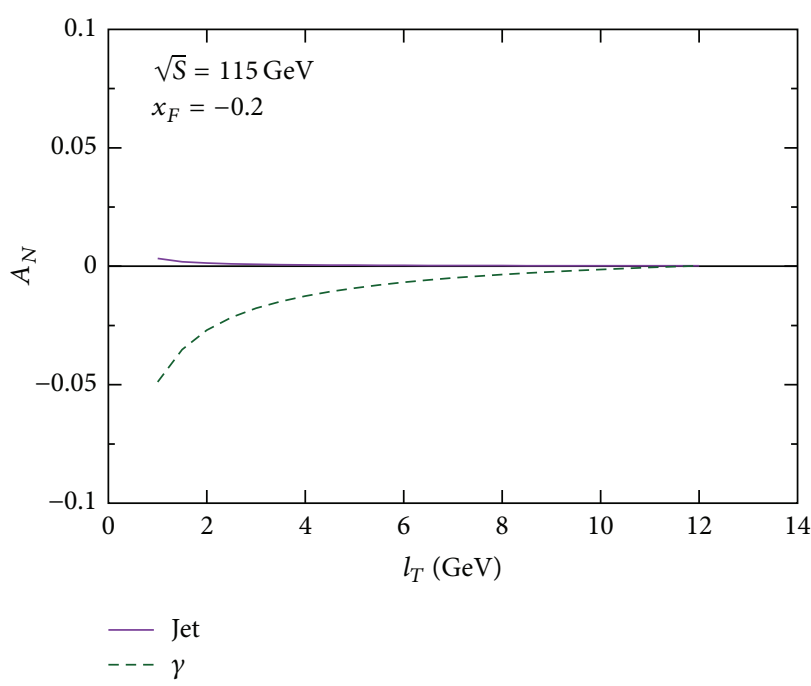

(a)

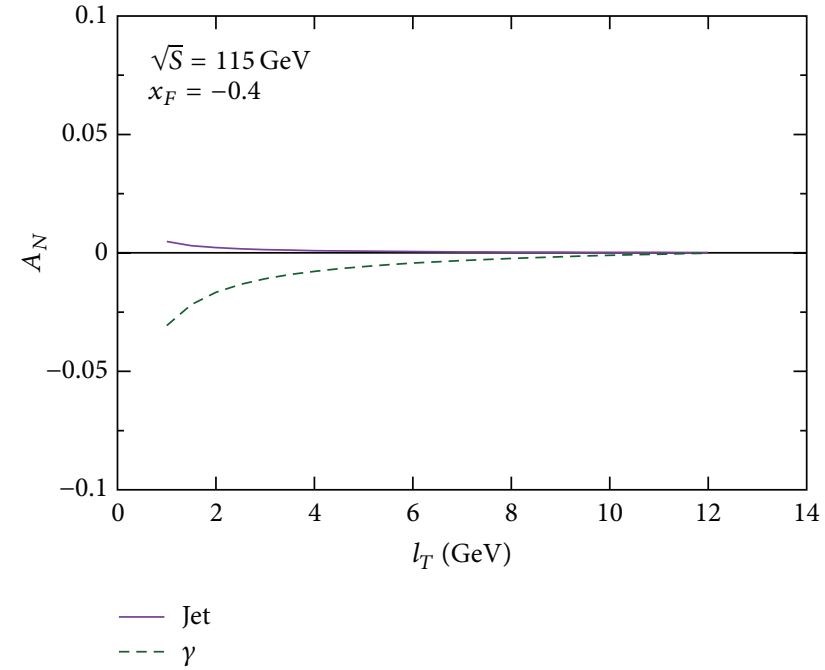

(b)

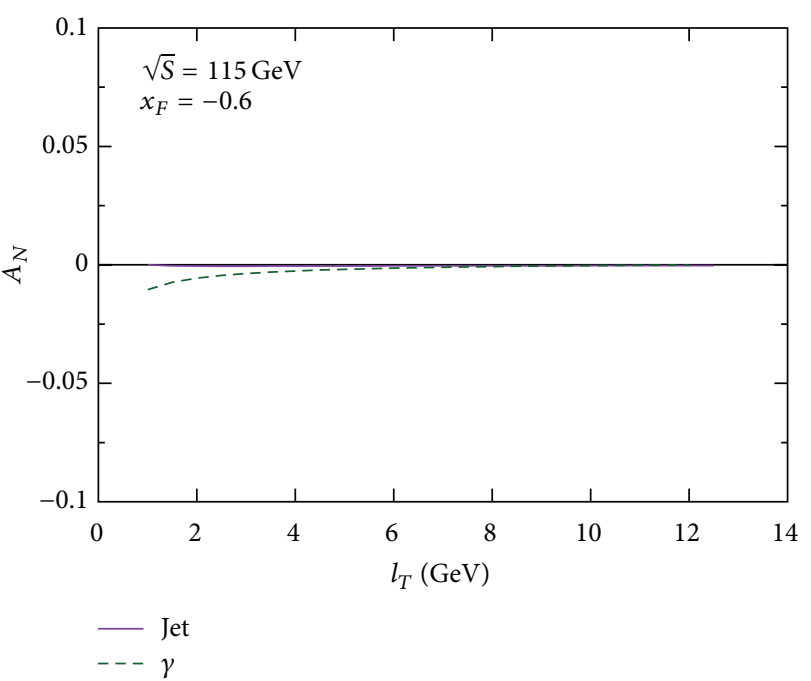

(c)

FIgURE 4: $A_{N}$ versus $l_{T}$ at fixed $x_{F}=-0.2(\mathrm{a}), x_{F}=-0.4(\mathrm{~b})$, and $x_{F}=-0.6(\mathrm{c})$ at $\sqrt{S}=115 \mathrm{GeV}$ for jet/photon production at AFTER.

if $A_{N}^{\pi}$ stays flat at higher- $l_{T}$, say to $12-15 \mathrm{GeV}$, like theory predicts $[29,51,55,59,70]$ and STAR has evidence for [71]. Also, higher statistics should allow for more precise measurements of $A_{N}^{\text {jet }}$ at more negative $x_{F}$, which will be necessary to determine if the QS function is the sole source of that asymmetry. Moreover, $A_{N}^{\gamma}$ has never been measured before and provides the opportunity to clearly distinguish between the CT3 and GPM frameworks and learn about the process dependence of the Sivers function. Given the questions that remain as to the origin of TSSAs, which has been unresolved for almost 40 years, AFTER could provide valuable data on these observables.

\section{Conflict of Interests}

The authors declare that there is no conflict of interests regarding the publication of this paper.

\section{Acknowledgments}

This work has been supported by the Grant-in-Aid for Scientific Research from the Japanese Society of Promotion of Science under Contract no. 26287040 (Y. Koike), the National Science Foundation under Contract no. PHY-1205942 (K. Kanazawa and A. Metz), and the RIKEN BNL Research Center (D. Pitonyak).

\section{References}

[1] G. Bunce, R. Handler, R. March et al., " $\Lambda 0$ hyperon polarization in inclusive production by 300 -gev protons on beryllium," Physical Review Letters, vol. 36, no. 19, pp. 1113-1116, 1976.

[2] G. L. Kane, J. Pumplin, and W. Repko, "Transverse quark polarization in large- $p_{T}$ reactions, $e^{+} e^{-}$jets, and leptoproduction: a test of quantum chromodynamics," Physical Review Letters, vol. 41, p. 1689, 1978 . 
[3] A. V. Efremov and O. V. Teryaev, "On spin effects in quantum chromodynamics," Soviet Journal of Nuclear Physics, vol. 36, p. 140, 1982, Engilsh translation to Yadernaya fizika, vol. 36, p. 242, 1982.

[4] A. V. Efremov and O. V. Teryaev, "QCD asymmetry and polarized hadron structure function measurement," Physics Letters B, vol. 150, no. 5, pp. 383-386, 1985.

[5] J.-W. Qiu and G. F. Sterman, "Single transverse spin asymmetries," Physical Review Letters, vol. 67, no. 17, pp. 2264-2267, 1991.

[6] J.-W. Qiu and G. F. Sterman, "Single transverse spin asymmetries in direct photon production," Nuclear Physics B, vol. 378, pp. 52-78, 1992.

[7] V. M. Korotkiyan and O. V. Teryaev, "Compton twist-three subprocesses for $p p$ single spin asymmetries," Physical Review D, vol. 52, no. 9, pp. R4775-R4779, 1995.

[8] J.-W. Qiu and G. F. Sterman, "Single transverse-spin asymmetries in hadronic pion production," Physical Review D, vol. 59, Article ID 014004, 1999.

[9] H. Eguchi, Y. Koike, and K. Tanaka, "Single transverse spin asymmetry for large- $p_{T}$ pion production in semi-inclusive deep inelastic scattering," Nuclear Physics B, vol. 752, no. 1-2, pp. 1-17, 2006.

[10] H. Eguchi, Y. Koike, and K. Tanaka, "Twist-3 formalism for single transverse spin asymmetry reexamined: semi-inclusive deep inelastic scattering," Nuclear Physics B, vol. 763, no. 1-2, pp. 198-227, 2007.

[11] C. Kouvaris, J. W. Qiu, W. Vogelsang, and F. Yuan, "Single transverse-spin asymmetry in high transverse momentum pion production in $p p$ collisions," Physical Review D, vol. 74, Article ID 114013, 2006.

[12] Y. Koike and K. Tanaka, "Master formula for twist-3 soft-gluonpole mechanism to single transverse-spin asymmetry," Physics Letters B, vol. 646, no. 5-6, pp. 232-241, 2007, Erratum in: Physics Letters B, vol. 668, no. 5, pp. 458-459, 2008.

[13] Y. Koike and K. Tanaka, "Universal structure of twist-3 softgluon-pole cross sections for single transverse-spin asymmetry," Physical Review D, vol. 76, no. 1, Article ID 011502(R), 5 pages, 2007.

[14] Y. Koike and T. Tomita, "Soft-fermion-pole contribution to single-spin asymmetry for pion production in $p p$ collisions," Physics Letters B, vol. 675, no. 2, pp. 181-189, 2009.

[15] K. Kanazawa and Y. Koike, "New analysis of the single transverse-spin asymmetry for hadron production at RHIC," Physical Review D, vol. 82, Article ID 034009, 2010.

[16] K. Kanazawa and Y. Koike, "A phenomenological study of single transverse-spin asymmetry for inclusive light-hadron productions at RHIC," Physical Review D, vol. 83, no. 11, Article ID 114024, 8 pages, 2011.

[17] K. Kanazawa and Y. Koike, "Contribution of the twist-3 fragmentation function to the single transverse-spin asymmetry in semi-inclusive deep inelastic scattering," Physical Review D, vol. 88, no. 7, Article ID 074022, 15 pages, 2013.

[18] A. Metz and D. Pitonyak, "Fragmentation contribution to the transverse single-spin asymmetry in proton-proton collisions," Physics Letters B, vol. 723, no. 4-5, pp. 365-370, 2013.

[19] H. Beppu, K. Kanazawa, Y. Koike, and S. Yoshida, “Three-gluon contribution to the single spin asymmetry for light hadron production in pp collision," Physical Review D, vol. 89, Article ID 034029, 2014.

[20] D. W. Sivers, "Single-spin production asymmetries from the hard scattering of pointlike constituents," Physical Review D, vol. 41, no. 1, pp. 83-90, 1990.
[21] D. Sivers, "Hard-scattering scaling laws for single-spin production asymmetries," Physical Review D, vol. 43, no. 1, pp. 261-263, 1991.

[22] J. Collins, "Fragmentation of transversely polarized quarks probed in transverse momentum distributions," Nuclear Physics $B$, vol. 396, no. 1, pp. 161-182, 1993.

[23] D. Boer and P. J. Mulders, "Time-reversal odd distribution functions in leptoproduction," Physical Review D, vol. 57, pp. 5780-5786, 1998.

[24] D. Boer and P. J. Mulders, "Investigating the origins of transverse spin asymmetries at BNL RHIC," Physical Review D, vol. 60, Article ID 014012, 1999.

[25] M. Anselmino, M. Boglione, and F. Murgia, "Single spin asymmetry for $p \uparrow p \rightarrow \pi X$ in perturbative QCD," Physics Letters B, vol. 362, no. 1-4, pp. 164-172, 1995.

[26] M. Anselmino and F. Murgia, "Single spin asymmetries in $p^{\uparrow} p$ and $\bar{p}^{\uparrow} p$ inclusive processes," Physics Letters B, vol. 442, no. 1-4, pp. 470-478, 1998.

[27] M. Anselmino, M. Boglione, U. D’Alesio, E. Leader, S. Melis, and F. Murgia, "General partonic structure for hadronic spin asymmetries," Physical Review D, vol. 73, Article ID 014020, 2006.

[28] M. Anselmino, M. Boglione, U. D’Alesio et al., "Role of collins effect in the single spin asymmetry $A_{N}$ in $p^{\uparrow} p \rightarrow h X$ processes," Physical Review D, vol. 86, Article ID 074032, 2012.

[29] M. Anselmino, M. Boglione, U. D’Alesio, S. Melis, F. Murgia, and A. Prokudin, "Sivers effect and the single spin asymmetry $A_{N}$ in $p^{\uparrow} p \rightarrow h X$ processes," Physical Review D, vol. 88, Article ID 054023, 2013.

[30] D. L. Adams, N. Akchurin, N. I. Belikov et al., "Comparison of spin asymmetries and cross sections in $\pi^{0}$ production by $200 \mathrm{GeV}$ polarized antiprotons and protons," Physics Letters B, vol. 261, no. 1-2, pp. 201-206, 1991.

[31] D. L. Adams, N. Akchurin, N. I. Belikov et al., "Analyzing power in inclusive $\pi^{+}$and $\pi^{-}$production at high $x_{F}$ with a $200 \mathrm{GeV}$ polarized proton beam," Physics Letters B, vol. 264, no. 3-4, pp. 462-466, 1991.

[32] K. Krueger, C. Allgower, T. Kasprzyk et al., "Large analyzing power in inclusive $\pi^{\neq}$production at high $x_{F}$ with a $22-\mathrm{GeV} / \mathrm{c}$ polarized proton beam," Physics Letters B, vol. 459, pp. 412-416, 1999.

[33] J. Adams, C. Adler, M. M. Aggarwal et al., "Cross sections and transverse single-spin asymmetries in forward neutral-pion production from proton collisions at $\sqrt{s}=200 \mathrm{GeV}$,' Physical Review Letters, vol. 92, Article ID 171801, 2004.

[34] B. I. Abelev, M. M. Aggarwal, Z. Ahammed et al., "Forward neutral-pion transverse single-spin asymmetries in $p+p$ collisions at $\sqrt{s}=200 \mathrm{GeV}$," Physical Review Letters, vol. 101, Article ID 222001, 2008.

[35] S. S. Adler, S. Afanasiev, C. Aidala et al., "Measurement of transverse single-spin asymmetries for midrapidity production of neutral pions and charged hadrons in polarized $p+p$ collisions at $\sqrt{s}=200 \mathrm{GeV}$,' Physical Review Letters, vol. 95, Article ID 202001, 2005.

[36] J. H. Lee, F. Videbæk, E. McBreen et al., "Single Spin Asymmetries of Identified Hadrons in Polarized $\mathrm{p}+\mathrm{p}$ at $\sqrt{s}=62.4$ and 200 GeV," AIP Conference Proceedings, vol. 915, p. 533, 2007.

[37] I. Arsene, I. G. Bearden, D. Beavis et al., "Single-transversespin asymmetries of identified charged hadrons in polarized $p p$ collisions at $\sqrt{s}=62.4 \mathrm{GeV}$,' Physical Review Letters, vol. 101, Article ID 042001, 2008. 
[38] L. Adamczyk, G. Agakishiev, M. M. Aggarwal et al., "Longitudinal and transverse spin asymmetries for inclusive jet production at mid-rapidity in polarized $p+p$ collisions at $\sqrt{s}=200 \mathrm{GeV}$," Physical Review D, vol. 86, Article ID 032006, 2012.

[39] L. Adamczyk, G. Agakishiev, M. M. Aggarwal et al., "Transverse single-spin asymmetry and cross section for $\pi^{0}$ and $\eta$ mesons at large Feynman $\mathrm{x}$ in $p^{\uparrow}+$ pcollisions at $\sqrt{s}=200 \mathrm{GeV}$," Physical Review D, vol. 86, Article ID 051101, 2012.

[40] L. C. Bland, E. Brash, H. J. Crawford et al., "Cross sections and transverse single-spin asymmetries in forward jet production from proton collisions at $\sqrt{s}=500 \mathrm{GeV}$," http://arxiv.org/abs/1304.1454.

[41] A. Adare, S. Afanasiev, C. Aidala et al., "Measurement of transverse-single-spin asymmetries for midrapidity and forward-rapidity production of hadrons in polarized $p+p$ collisions at $\sqrt{s}=200$ and $62.4 \mathrm{GeV}$," Physical Review D, vol. 90, Article ID 012006, 2014.

[42] A. Adare, C. Aidala, N. N. Ajitanand et al., "Cross section and transverse single-spin asymmetry of $\eta$ mesons in $p^{\uparrow}+p$ collisions at $\sqrt{s}=200 \mathrm{GeV}$ at forward rapidity," Physical Review D, vol. 90, Article ID 072008, 2014.

[43] J. Nagle, PHENIX Beam Use Proposal: Run-15 and Run-16, University of New Mexico, 2014.

[44] The STAR Collaboration, "RHIC Beam Use Request for Runs 15 and 16," June 2014.

[45] E.-C. Aschenauer, A. Bazilevsky, M. Diehl et al., "The RHIC SPIN program: achievements and future opportunities," http:// arxiv.org/abs/1501.01220.

[46] Z.-B. Kang, J.-W. Qiu, W. Vogelsang, and F. Yuan, "Observation concerning the process dependence of the Sivers functions," Physical Review D, vol. 83, Article ID 094001, 2011.

[47] Z.-B. Kang and A. Prokudin, "Global fitting of single spin asymmetry: an attempt," Physical Review D, vol. 85, Article ID 074008, 2012.

[48] A. Metz, D. Pitonyak, A. Schafer, M. Schlegel, W. Vogelsang, and J. Zhou, "Single-spin asymmetries in inclusive deep inelastic scattering and multiparton correlations in the nucleon," Physical Review D, vol. 86, Article ID 094039, 2012.

[49] A. Airapetian, N. Akopov, Z. Akopov et al., "Search for a two-photon exchange contribution to inclusive deep-inelastic scattering," Physics Letters B, vol. 682, pp. 351-354, 2010.

[50] J. Katich, X. Qian, Y. X. Zhao et al., "Measurement of the targetnormal single-spin asymmetry in deep-inelastic scattering from the reaction ${ }^{3} \mathrm{He}^{\uparrow}\left(e, e^{\prime}\right) X$," Physical Review Letters, vol. 113, Article ID 022502, 2014.

[51] K. Kanazawa, Y. Koike, A. Metz, and D. Pitonyak, "Towards an explanation of transverse single-spin asymmetries in protonproton collisions: the role of fragmentation in collinear factorization," Physical Review D, vol. 89, Article ID 111501, 2014.

[52] Z. B. Kang, F. Yuan, and J. Zhou, "Twist-three fragmentation function contribution to the single spin asymmetry in $p p$ collisions," Physics Letters B, vol. 691, pp. 243-248, 2010.

[53] L. Gamberg, Z. B. Kang, A. Metz, D. Pitonyak, and A. Prokudin, "Left-right spin asymmetry in $\ell N^{\uparrow} \rightarrow h X$, , Physical Review D, vol. 90, Article ID 074012, 2014.

[54] K. Kanazawa, A. Metz, D. Pitonyak, and M. Schlegel, "Longitudinal-transverse double-spin asymmetries in singleinclusive leptoproduction of hadrons," Physics Letters B, vol. 742, pp. 340-346, 2015.

[55] M. Anselmino, U. D’Alesio, and S. Melis, “Transverse single-spin asymmetries in proton-proton collisions at the
AFTER@LHC experiment in a TMD factorisation scheme," http://arxiv.org/abs/1504.03791.

[56] L. Gamberg and Z. B. Kang, "Single transverse spin asymmetry of prompt photon production," Physics Letters B, vol. 718, no. 1, pp. 181-188, 2012.

[57] Y. V. Kovchegov and M. D. Sievert, "New mechanism for generating a single transverse spin asymmetry," Physical Review $D$, vol. 86, Article ID 034028, 2012, Erratum in: new mechanism for generating a single transverse spin asymmetry, Physical Review D, vol. 86, Article ID 079906, 2012.

[58] A. Schafer and J. Zhou, "Transverse single spin asymmetry in direct photon production in polarized $p A$ collisions," Physical Review D, vol. 90, Article ID 034016, 2014.

[59] K. Kanazawa and Y. Koike, "Single transverse-spin asymmetry for direct-photon and single-jet productions at RHIC," Physics Letters B, vol. 720, pp. 161-165, 2013.

[60] L. Gamberg, Z. B. Kang, and A. Prokudin, "Indication on the process dependence of the sivers effect," Physical Review Letters, vol. 110, Article ID 232301, 2013.

[61] X. Ji, J. W. Qiu, W. Vogelsang, and F. Yuan, "Single transversespin asymmetry in Drell-Yan production at large and moderate transverse momentum," Physical Review D, vol. 73, Article ID 094017, 2006.

[62] Y. Koike and S. Yoshida, "Three-gluon contribution to the single spin asymmetry in Drell-Yan and direct-photon processes," Physical Review D, vol. 85, Article ID 034030, 2012.

[63] K. Kanazawa and Y. Koike, "Hard- and soft-fermion-pole contributions to single transverse-spin asymmetry for DrellYan process," Physics Letters B, vol. 701, pp. 576-580, 2011.

[64] K. Kanazawa, Y. Koike, A. Metz, and D. Pitonyak, "Transverse single-spin asymmetries in $p^{\uparrow} p \rightarrow \gamma X$ from quark-gluonquark correlations in the proton," Physical Review D, vol. 91, Article ID 014013, 2015.

[65] J. C. Collins, "Leading-twist single-transverse-spin asymmetries: Drell-Yan and deep-inelastic scattering," Physics Letters B, vol. 536, no. 1-2, pp. 43-48, 2002.

[66] Y. Kanazawa and Y. Koike, "Chiral-odd contribution to singletransverse spin asymmetry in hadronic pion production," Physics Letters B, vol. 478, pp. 121-126, 2000.

[67] Y. Kanazawa and Y. Koike, "Estimate of a chiral-odd contribution to single transverse-spin asymmetry in hadronic pion production," Physics Letters B, vol. 490, pp. 99-105, 2000.

[68] S. J. Brodsky, F. Fleuret, C. Hadjidakis, and J. P. Lansberg, "Physics opportunities of a fixed-target experiment using LHC beams," Physics Reports, vol. 522, no. 4, pp. 239-255, 2013.

[69] J. P. Lansberg, M. Anselmino, R. Arnaldi et al., "Spin physics and TMD studies at a fixed-target experiment at the LHC (AFTER@LHC),"EPJ Web of Conferences, vol. 85, Article ID 02038, 2015.

[70] M. Anselmino, D. Boer, U. D’Alesio, and F. Murgia, “ $\Lambda$ polarization from unpolarized quark fragmentation," Physical Review $D$, vol. 63, Article ID 054029, 2001.

[71] S. Heppelmann, PoS DIS, vol. 2013, p. 240, 2013.

[72] D. Boer and C. Pisano, "Polarized gluon studies with charmonium and bottomonium at LHCb and AFTER," Physical Review $D$, vol. 86, Article ID 094007, 2012.

[73] A. Schäfer and J. Zhou, "Transverse single spin asymmetry in hadronic $\eta_{c, b}$ production," Physical Review D, vol. 88 , no. 1 , Article ID 014008, 2013. 
[74] D. Boer, P. J. Mulders, and F. Pijlman, "Universality of Todd effects in single spin and azimuthal asymmetries," Nuclear Physics B, vol. 667, pp. 201-241, 2003.

[75] M. M. Mondal et al., "Measurement of the transverse singlespinasymmetries for $\pi^{0}$ and jet-like events at forward rapidities at STAR in $p+p$ Collisions at $\sqrt{s}=500 \mathrm{GeV}$,' Proceedings of Science, vol. DIS2014, p. 216, 2014, http://arxiv.org/abs/1407.3715. 

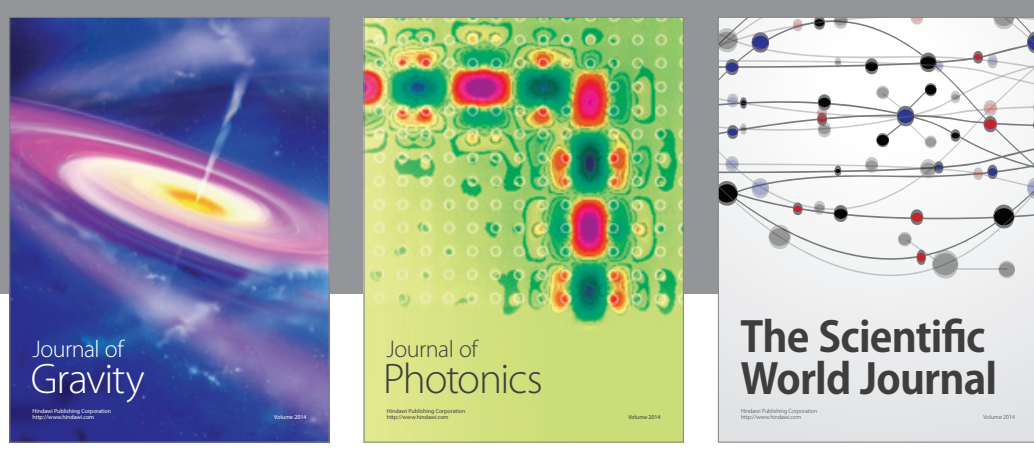

The Scientific World Journal
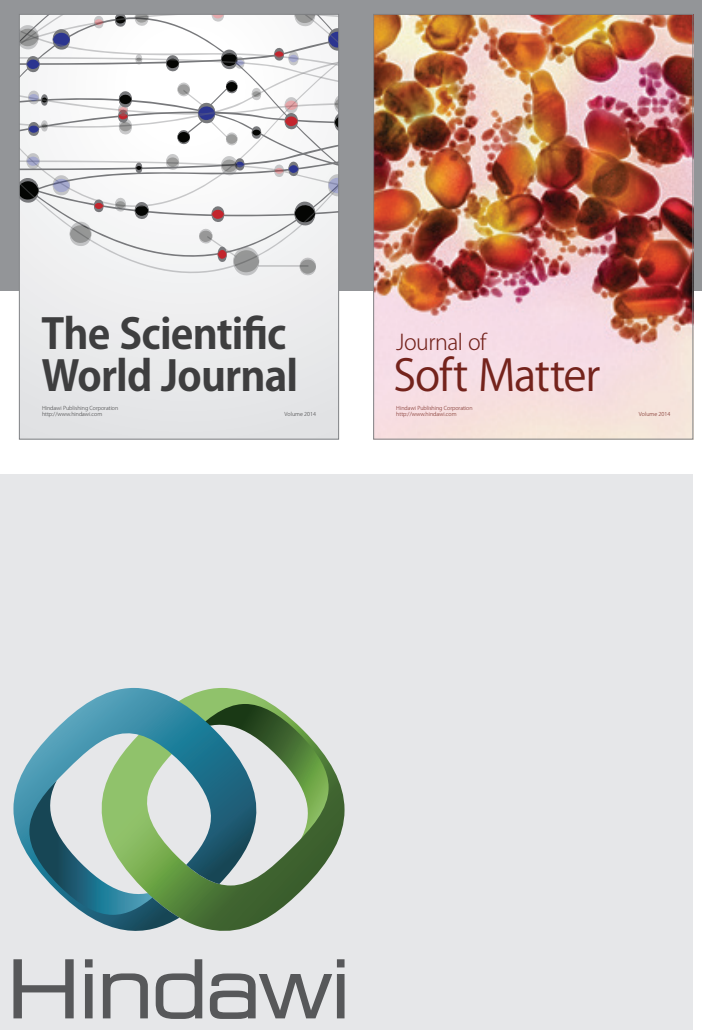

Submit your manuscripts at

http://www.hindawi.com

nternational Journal of

Statistical Mechanics
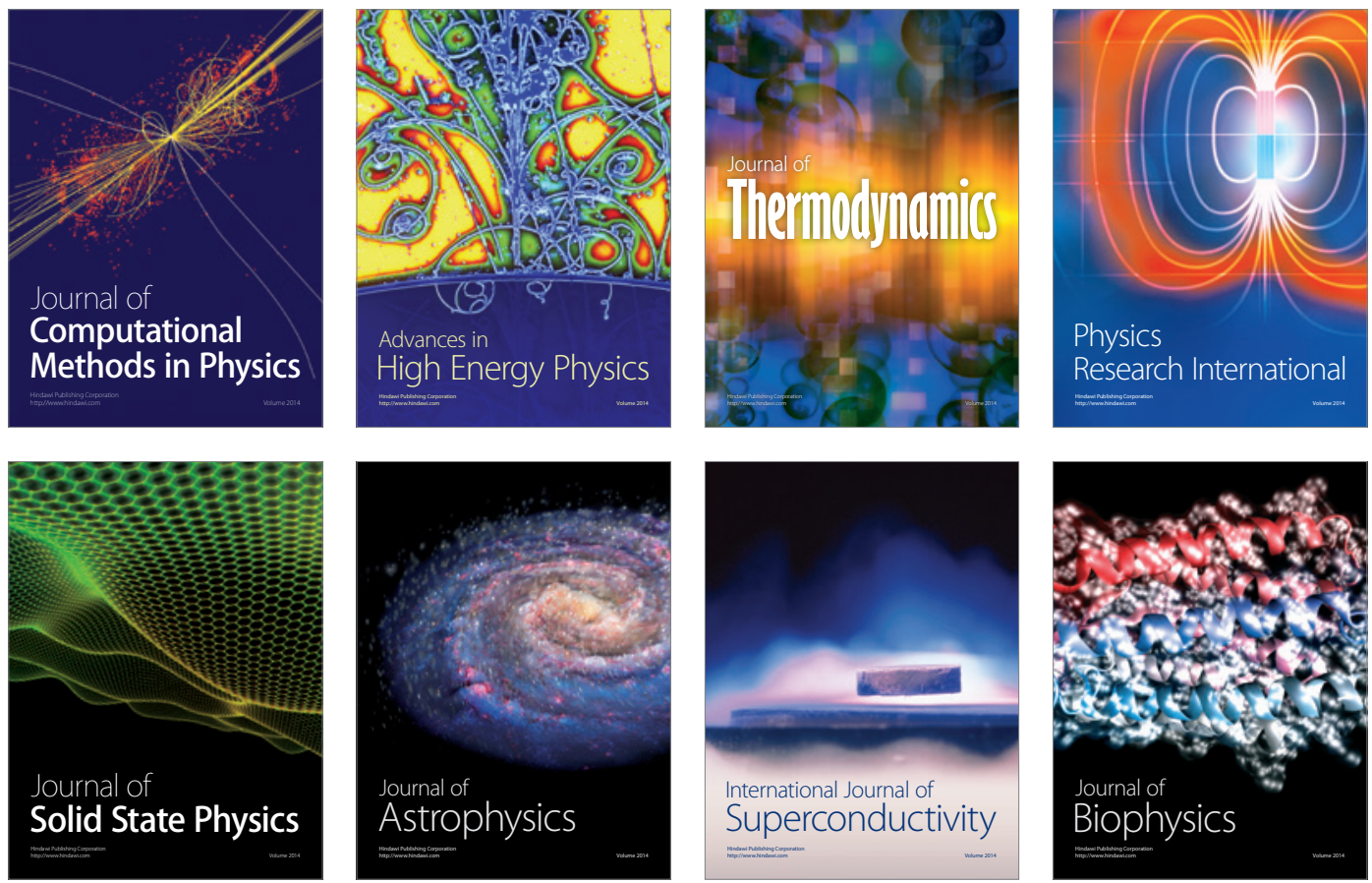
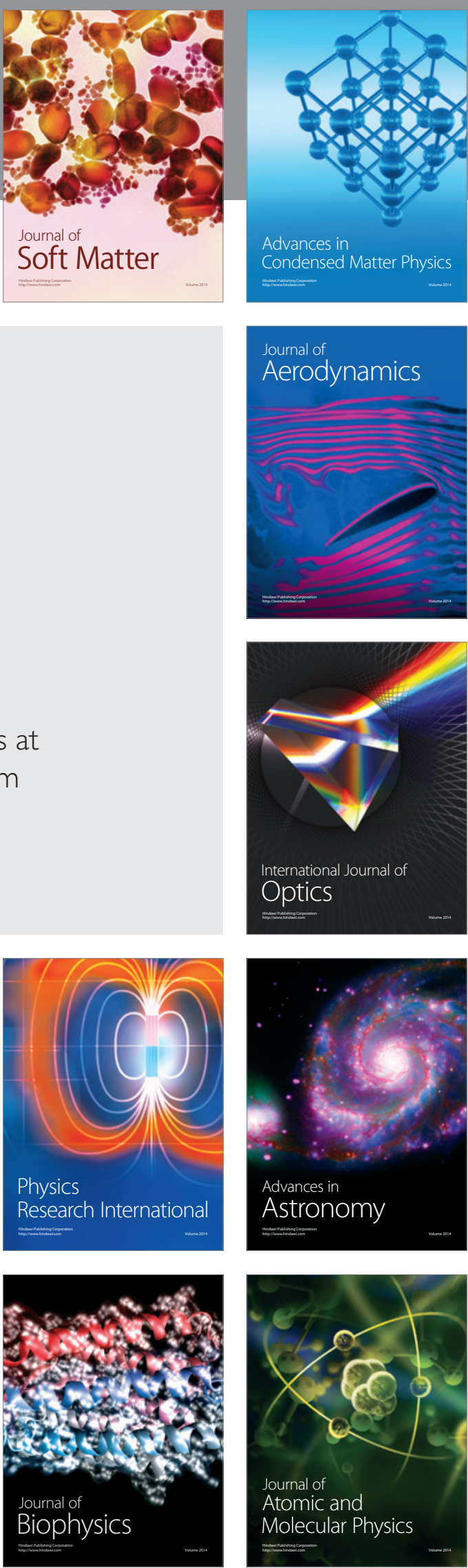